\title{
Nutritional Vitamin D Deficiency Rickets
}

National Cancer Institute

\section{Source}

National Cancer Institute. Nutritional Vitamin D Deficiency Rickets. NCI Thesaurus. Code C131448.

Nutritional rickets due to dietary deficiency of vitamin D. 